\title{
La construcción musical temporal interpersonal en la fila de orquesta
}

\author{
Juliette Epele' e Isabel Cecilia Martínez² \\ epelejuliette@gmaill.com \\ Laboratorio para el Estudio de la Experiencia Musical - Facultad de Artes \\ Universidad Nacional de La Plata.
}

\section{Resumen}

En este trabajo se estudia la construcción temporal de un fragmento musical en una situación espontánea de reinterpretación en fila de orquesta. Tres violistas de fila fueron convocadas para ensamblar en vivo un fragmento musical de 13 compases de la sección introductoria del tercer movimiento de "Mathis der Maler" de Paul Hindemith, en la que el orgánico de cuerdas interpreta al unísono y octava. Una vez completada la tarea, las ejecutantes resolvieron rehacer espontáneamente el compás final correspondiente a la cadencia y cierre del fragmento. Se analizó la variabilidad horizontal y vertical de intervalos de tiempo entre ataques sonoros (ITEA) comparativamente entre la versión inicial y la reinterpretación de dicho final. El análisis de la interacción entre ejecutantes fue abordado en base a las nociones líder/seguidor. Los resultados muestran una elevada sincronización entre intérpretes. Las diferencias entre ITEA entre versiones evidencian una participación activa de las violistas para alcanzar un toque al unísono, independientemente de los roles asignados. Notoriamente, la variabilidad temporal entre intérpretes se redujo al mínimo a la vez que la sincronización aumentó en puntos de inflexión relativos a la estructura métrica de la música. El alineamiento detrás de la voz del guía convive con el anclaje métrico como forma organizativa de la ejecución. Como surge de las entrevistas, la construcción temporal unívoca en fila de orquesta sugiere una acción continua e interdependiente de microajustes y compensaciones momento a momento, de naturaleza adaptativa, incluyendo la conducta flexible, dinámica y colaborativa del guía, quien participa activamente del objetivo común.

\section{Palabras Clave}

Ejecución expresiva, alineamiento interpersonal, variabilidad, sincronización adaptativa, guía/seguidor. 


\title{
Musical interpersonal time shaping at the orchestral line
}

\begin{abstract}
This work studies the temporal construction of a musical fragment in the orchestra row in a spontaneous reinterpretation trail during rehearsal. Three orchestral row violists were summoned to assemble live a 13-bar musical fragment from the introductory section of Paul Hindemith's 3rd movement "Mathis der Maler", in which the string orchestra plays in unison and eighths. Once the task was completed, the performers resolved to spontaneously replay the final section corresponding to the cadence and closure of the fragment. The horizontal and vertical variability of the time intervals between sound attacks (IOI) was analyzed comparatively between the initial version and the reinterpretation of the above-mentioned end. The analysis of the interaction between performers was approached based on the notions of leader/follower. The results show a high-level synchronization between performers in both versions. Differences in the IOIs between versions show an active participation of the violists to achieve a unison sound, regardless of the assigned guide/follower roles. Notably, the temporal variability between performers was minimized at metric structural locations, while, at the same time the synchrony between performers increased. Alignment behind the leader voice cohabit metrical anchoring as a way of organizing performance. As emerges from the interviews carried out, the univocal temporal construction in an orchestra row suggests a continuous and interdependent action of moment-by-moment micro-adjustments and compensations, adapting performance both to the leader's voice and to each other. It also includes the flexible, dynamic, and collaborative behavior of the guide, who actively participates in the common objective.
\end{abstract}

\section{Key Words}

Expressive performance, interpersonal alignment, variability, adaptative timing, leader/ follower

\section{Introducción}

La actividad musical de conjunto es un asunto típicamente colectivo que implica la participación activa en la comunión de intenciones expresivas, compartidas y comunicadas entre co-participantes y al público en tiempo real (De Jaegher y Di Paolo, 2007). Esta actividad encuentra sus bases en las interacciones comunicativas tempranas entre adultos de crianza y bebés, cuya musicalidad satisface el mutuo deseo de acompañarse el uno al otro y dialogar, intercambiando dinámica y bidireccionalmente frases de sonido y movimiento corporal gestual, desarrolladas 
por turnos o de manera superpuesta y acoplada en el tiempo (Malloch y Trevarthen, 2009; Español y Shifres, 2015; Martínez, 2014). El desarrollo y refinamiento de las formas expresivas de la interpretación conjunta, aún en los más altos niveles de la práctica del arte musical experto, suponen -por lo tanto- una construcción dinámica basada en el intercambio de los gestos expresivos con las otras personas interactuantes (Leman, 2016), en el caso particular de la fila de orquesta, con el objeto de sonar como si todos fueran una misma voz. En razón de esto, las formas interactivas exponen como requisito fundamental el alineamiento interpersonal (Leman, 2016) de la gestualidad musical y corporal, implicando la realización de continuos ajustes temporales individuales a fin de lograr el acople y mantener el tiempo y la sincronía en y con el grupo, dadas las microviariaciones expresamente motivadas o aleatorias relativas a la ejecución expresiva (Palmer, 1997).

Las variaciones de tiempo referidas a la interpretación musical comprometen las relaciones sucesivas, horizontales y verticales, o sincrónicas entre ataques de sonido, creando respecto de la ejecución en conjunto un objetivo de sincronización móvil (Keller, 2014) díficilmente preciso cuando las líneas melódicas idénticas pretenden a su vez correr en paralelo. De este modo se ponen en acto mecanismos capaces de sustentar la interacción, permitiendo anticipar, reaccionar y asistir a las señales multisensoriales generadas en el transcurso de la co-interpretación. La activación de relojes internos flexibles generadores de intervalos o de patrones oscilatorios (Keller, 2008) posibilita el control de aspectos temporales y la sincronización a través de la corrección de errores de fase o de período, propios y/o entre co-ejecutantes, y la asimilación y reproducción de formas gestuales concurrentes (Keller, 2014), que operan tanto a niveles automático como consciente. No obstante, el uso de imágenes anticipatorias (Keller, 2008) facilita la predicción de eventos y la planificación y calibración de modelos internos propios al estilo de acción de los otros, sobre la base de procesos de simulación del comportamiento y de información aportada por retroalimentación sensoriomotora continua. Los esquemas predictivos sensoriomotores subyacen a la interacción gestual basada en la generación y el alineamiento de patrones expresivos biosociales (Leman, 2016), favoreciendo una actividad motivacionalmente gratificante fundada en el sentimiento de mutua agencia de los participantes con la música. La agencia surge precisamente cuando las predicciones coinciden con las entradas sensoriales y los esfuerzos físicos interindividuales logran aunarse sostenidamente en el tiempo. Sin embargo, puede verse igualmente inducida por situaciones de interacción no controladas, subordinada al impulso de aspectos intencionales, energéticos y afectivos pro-sociales que convierten el flujo sensorial en modelos predictivos de acción participativa con el otro y conducen a los sentimientos de poder, unidad e inmersión (Clarke, 2014). 
Uno de los factores que contribuyen al logro del alinemiento expresivo en la actividad rítmica de la fila de orquesta refiere a la sofisticada capacidad que logran los músicos para ajustar la periodicidad en la generación de patrones de ejecución motora a la inducción o a la extracción de las diferentes unidades y niveles de pulsación que integran la jerarquía métrica de cualquier pieza musical (Lerdahl y Jackendoff, 1983). Y es por medio de la sincronización o el entrainement de la propia actividad motora con la pulsación inferida, que dicha capacidad es puesta en juego simultáneamente en la sincronización o el entrainement con la generación de patrones de los ejecutantes. Se ha sugerido que la isocronicidad en tanto disposición básica humana resulta de la interacción entre la extracción de los patrones de pulsación y una motivación o deseo, de base adaptativa-evolutiva, por el logro o la obtención de la coherencia intersubjetiva grupal (Tecumseh Fitch, 2012). De ahí que la extracción del pulso deviene una actividad cognitiva básica que se torna crucial para la realización de acciones sofisticadas de ejecución coarticuladas en el conjunto instrumental musical (Lerdahl y Jackendoff, 1983), dada la potencialidad que tiene la música para ofrecer una organización métrica multinivel a la cognición auditiva y con ello devenir en medio apto para la conducta sincrónica, susceptible de convertir los eventos sonoros emergentes de los patrones de acentuación multinivel en anclas para la organización tanto del entrainement performativo como de la anticipación del devenir temporal de la ejecución (Repp, 2010; Madison, Ullén y Merker, 2017). En tal sentido, los marcos métricos se comportan como marcos cognitivo-motores capaces de facilitar la percepción rítmica y la acción, alentando la asignación de recursos atencionales integradores priorizados (Keller, 2008) de acuerdo con las periodicidades de la estructura musical subyacente. Adicionalmente, el alineamiento temporal entre los intérpretes se redimensiona a partir de las claves visuales que brindan las marcas discretas de tiempo asociadas a los patrones de movimiento espacial, emergentes de la gestualidad de los intérpretes (Morán, 2017).

Luego, en ausencia del director los músicos asumen roles más o menos intercambiables. La asignación de los roles líder/seguidor puede estar dada por la regularidad y la división o subdivisión rítmica de las partes individuales (Goebl y Palmer, 2009; Varni, Volpe y Camurri, 2010). Pero cuando las partes musicales son idénticas -como ocurre en una fila de orquesta- entran en juego factores tales como la personalidad y la experticia de cada uno de los integrantes del grupo (Keller, 2014). Las estrategias pueden variar desde en seguimiento obediente hasta la participación de todos los miembros actuando y reaccionando entre sí como equipo, dinámica y mutuamente, autogestionado y autorganizado (Goodman, 2002; Timmers, Endo, Bradbury y Wing, 2014), un fénomeno explicable sólo en los términos de la propia emergencia colaborativa (Sawyer, 2006). Los estudios sobre variabilidad temporal en conjunto sugieren mayor imprecisión y adaptación por 
parte del seguidor, siendo claves la información auditiva con importante asistencia de la información visual (Goebl y Palmer, 2009; Keller y Appel, 2010). El lapso entre la percepción del sonido del otro y la producción del propio normaliza un asincronismo entre ataques de sonido por encima del umbral perceptivo (Goebl y Parncutt, 2002) en el orden de los 30 a $50 \mathrm{~ms}$ (Rasch, 1979), en principio menor de lo que se esperaría de la reacción de un músico en calidad de seguidor frente a un líder (Keller, 2008). Si, en cambio, se ignoran las relaciones líder/seguidor, la separación promedio entre sonidos nominalmente sincrónicos puede rozar incluso los 200 ms o más (Keller, 2014). Se ha comprobado que los sonidos iniciales de frase, en especial aquellos precedidos de largo silencio, resultan particularmente díficiles de cosincronizar (Rasch, 1979), y que la temporización vertical se aplica sistemáticamente y se correlaciona con el desempeño musical repetido (Shaffer, 1984). A propósito, estudios sobre percepción de la regularidad temporal en las actuaciones musicales sugieren que la misma se basa en expectativas que se adaptan a entradas temporalmente fluctuantes, respecto de las que se observan el surgimiento de patrones de asincronía cualitativamente diferentes dependiendo del nivel métrico que sirva de objetivo a la sincronización (Large y Palmer, 2002), con una mayor precisión en los niveles métricos más altos que en los más bajos (Rankin, Large y Fink, 2009).

Si bien los estudios acerca de la sincronización entre intérpretes durante la performance de conjunto a los que se ha hecho referencia aquí brindan información relevante en cuanto a la regulación temporal y la dependencia entre los ejecutantes, los análisis anteriores han abordado poco la complejidad resultante de la sincronización entre intérpretes cuando la superficie rítmico-métrica y temporal presenta un alto grado de variabilidad. El presente estudio cumple el propósito de utilizar las técnicas del análisis temporal de la ejecución y de aplicarlas en el estudio de la sincronización entre ejecutantes al abordar la interpretación expresiva de una superficie musical con este tipo de complejidad temporal.

\section{Objetivo}

El presente trabajo se propone analizar comparativamente la construcción temporal de un fragmento musical en una situación espontánea de reinterpretación en fila de orquesta, respecto del que los ejecutantes mostraron mayor conformidad en relación a su segunda versión. En particular serán objetivos específicos: (a) describir y analizar comparativamente el comportamiento temporal horizontal y vertical entre versiones, y (b) analizar la interacción entre los ejecutantes relativa a la construcción conjunta respecto de las nociones de líder/seguidor. 


\section{Metodología}

\section{Participantes}

Participaron del experimento 3 violistas mujeres jóvenes de 22, 25 y 44 años de edad $(M=30,33)$, graduadas, ejecutantes profesionales e integrantes de distintas orquestas de la ciudad de La Plata y de la Ciudad Autónoma de Buenos Aires, con 4, 10 y 20 de experiencia respectivamente $(M=11,33)$.

\section{Estímulo musical}

El fragmento musical propuesto como estímulo consistió en los 13 compases de la sección introductoria "Sehr Langsam, frei im Zeitmaß" del tercer movimiento de "Mathis der Maler" de Paul Hindemith, un extracto en el que el orgánico de cuerdas de la orquesta interpreta al unísono y octava, en contraposición a otros grupos, una melodía de carácter improvisatorio de base cromática y métrica irregular marcada por indicación de tiempo libre, cambios de compás, valores irregulares, calderones, rubato y una amplia variedad de valores rítmicos comprendidos entre la fusa y la blanca con 4 puntillos. El fragmento forma parte del repertorio orquestal conocido por las participantes y los músicos de orquesta en general.

\section{Aparatos}

Para el registro de audio se utilizaron micrófonos condenser Rode NT-1 y paneles acústicos aislantes portátiles. La toma se transfirió por separado y guardó con Logic Studio Pro en una PC de escritorio (Apple, CA, EE.UU.) y los archivos WAV sin comprimir se analizaron con Sonic Visualiser 3.2 (Chris Cannam, Queen Mary University of London) y Stata 13.

\section{Procedimiento}

Los músicos fueron convocados para el ensamble en vivo del fragmento musical propuesto e indicado con anticipación a su encuentro, para lo cual se les solicitó no realizar ensayos ni prácticas conjuntas previas al inicio del registro, a desarrollarse en una sola toma. Una vez completada la tarea y sin solicitar detener ni corregir el registro, sin embargo, las ejecutantes continuaron y resolvieron rehacer, sin especificaciones, el compás final correspondiente a la cadencia y cierre del fragmento musical convenido. Ese fue precisamente el tramo del registro que motivó y que -bajo consentimiento de los músicos- fue tomado a los fines de la presente investigación.

El procedimiento de grabación tuvo lugar en un estudio en el que la dispo- 
sición espacial de las participantes privilegió su total y cómoda visualización, previendo la necesidad de aislar el sonido mediante paneles acústicos portátiles.

Una vez finalizado el registro se procedió a entrevistar individualmente a las participantes acerca de su experiencia respecto de: (a) su concepción de la sincronización en la fila de orquesta; (b) su concepción de la sincronización en la experiencia particular; (c) la urgencia de mirarse y/o escucharse durante la tarea, u otras estrategias ante la ausencia de director; y (d) su opinión respecto de la reinterpretación.

\section{Resultados}

\section{Variabilidad temporal y asincronismo durante la ejecución}

A partir de las diferencias de duración entre la ejecución primera $(5,979$ s) y la segunda (7,436 s) se examinó la variabilidad del intervalo de tiempo entre ataques de sonidos sucesivos normalizados (ITEA) relativo al valor nominal de la corchea en tanto valor mediano referente. Los resultados sobre variabilidad horizontal registran un promedio de duración de la corchea de 1,252 s en la primera versión y 1,369 s en la segunda, con una mayor amplitud entre valores mínimo y máximo en el segundo caso, lo que da cuenta que los distintos valores rítmicos del fragmento musical fueron interpretados más lento y con una mayor variabilidad en su segunda versión (ver Tabla 1).

\begin{tabular}{cccccc}
\hline Variable & Obs & SD & Mean & Min & Max \\
\hline Ejecución 1 & 30 & .0903051 & 1.251976 & 1.067282 & 1.436671 \\
Ejecución 2 & & .0990235 & 1.368854 & 1.166428 & 1.57138
\end{tabular}

Tabla 1. Valores promedio, minimo y máximo del valor nominal referente (corchea) de cada una de las ejecuciones (intervalo de confianza del 95\%).

En razón de las diferencias encontradas, se procedió a contrastar y a analizar las variaciones temporales evento por evento a cargo de cada uno de las ejecutantes en una y otra interpretación. Al respecto, la Figura 1 representa las diferencias punto por punto, donde los valores positivos indican alargamiento del ITEA en la segunda versión con relación a la primera, y visceversa. De este modo, se observa que (a) los ITEA sufren variaciones no iguales de una versión a otra; (b) el apartamiento positivo o negativo del ITEA es independiente del valor de la unidad rítmica escrita (por ejemplo, eventos 1 al 5); (c) la magnitud del ITEA 
varía según el participante (por ejemplo, el evento 8 debió alargarse para la viola 2 , en tanto que las violas 1 y 3 lo acortaron respecto de su primera versión); (d) las variaciones son algunas veces más acentuadas en la viola 1 y otras en la viola 2 o en la viola 3; y (e) los eventos con menor variación (eventos 3 y 6) corresponden a la unidad de articulación de corchea. Este comportamiento describe una recreación musical no debida a las unidades rítmicas escritas, sino a la versión de la que participan las tres ejecutantes por igual, haciendo continuos ajustes. Por otra parte, los acortamientos o alargamientos eventuales según la versión no parecen tener el mismo grado de variación respecto de su ubicación en la estructura musical, siendo los eventos producidos en la división de la unidad de pulso los que registran mínima variación tanto entre versiones como entre participantes.

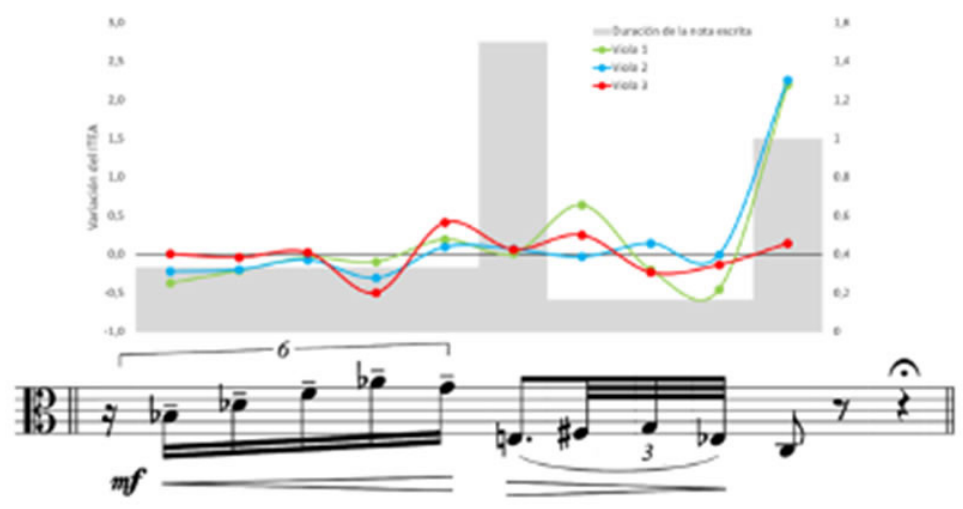

Figura 1. Diferencias de alargamiento (valor positivo) o acortamieno (valor negativo) del ITE $A$ normalizado en segundos (eje izquierdo) de cada participante entre versiones. En gris se representa la duración de la unidad rítmica escrita, donde $1=$ corchea (eje derecho).

A continuación, se calculó el asincronismo evento por evento por cada par de ejecutantes, observándose una correlación positiva elevada en todos los pares (Tabla 2), no llamativa en una ejecución experta que busca la unidad. Luego se analizó -a partir de los valores en bruto- el grado de alineamiento de los músicos, advirtiendo que la viola 3 aparecía por delante de las otras dos violas en un 50\% de los ataques en la primera ejecución y en un $90 \%$ en la segunda, en la que el par de violas 1 y 2 alcanzó, a su vez, el asincronismo medio más pequeño $(12 \mathrm{~ms}$, contra los $42 \mathrm{~ms}$ promedio alcanzados durante la primera interpretación). Los restantes pares de ejecutantes asumieron valores levemente más altos, promediando un máximo de 79 ms entre viola 1 y viola 3 en la reinterpretación. No obstante, 
durante la primera versión -excepcionalmente- el par viola 2-viola 3 obtuvo un promedio de $9 \mathrm{~ms}$ en valores positivos, lo que significó que la viola 2 sonó relativamente por delante respecto de la viola guía o viola 3.

\begin{tabular}{cccc} 
& Violas 1/2 & Violas 2/3 & Violas 1/3 \\
\hline Ejecución 1 & 0,99974982 & 0,99943373 & 0,99887124 \\
Ejecución 1 & 0,99984071 & 0,99979422 & 0,99965807
\end{tabular}

Tabla 2. Coeficientes de correlación por pares de ejecutantes. Primera y segunda ejecución.

Finalmente, un análisis de las diferencias normalizadas entre los ataques más aventajado y atrasado de la producción de cada sonido en ambas versiones (Figura 2) mostró una tendiente reducción del asincronismo a partir del tercer sonido, probablemente porque los músicos ya venían tocando tras el comienzo acéfalo, como también un aumento hacia el final, especialmente en la segunda versión donde tienen lugar el cierre y decaimiento de la frase musical construido sobre un tresillo de fusas. Los valores de separación normalizados entre ataques nominalmente sincrónicos alcanzaron un máximo de $0,682 \mathrm{seg}$. No obstante, evidenciaron cambio y reducción en relación con la estructura temporal, otra vez, sobre la división del pulso (evento 3) y la pulsación (eventos 6 y 10), siendo los mínimos valores de separación promedio o asincronismo registrados: $0,237 \mathrm{~s}, 0,020 \mathrm{~s}$ y $0,124 \mathrm{~s}$ para la primera versión, y $0,277 \mathrm{~s}, 0,044$ s y $0,115 \mathrm{~s}$ para la segunda. Considérese el primer pulso a tierra o battere, en silencio.

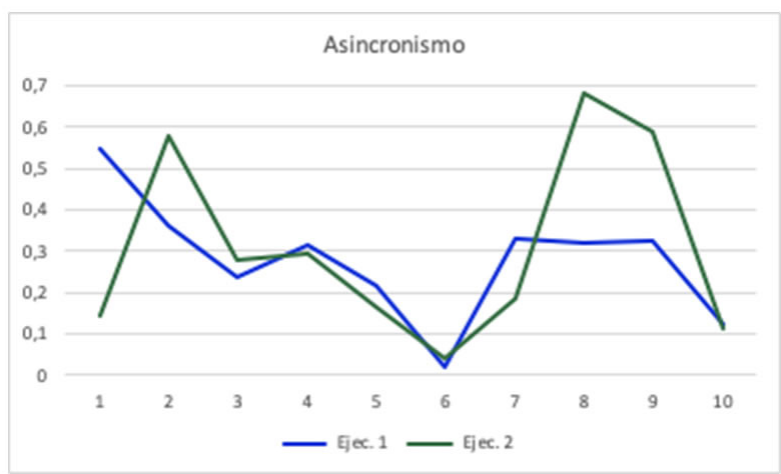

Figura 2. Separación temporal promedio entre ataques de sonido nominalmente sincrónicos en segundos de la primera y la segunda ejecución. 


\section{Experiencia interactiva informada por los ejecutantes}

Las observaciones referidas al alineamiento temporal tanto expresivo como sincrónico se compararon con las ideas y las estrategias reportadas por las ejecutantes. $\mathrm{Al}$ respecto, la presencia del guía y la atención compartida adquirieron particular relevancia tanto en lo que hace a la experiencia general en la fila como a su concreta participación en la presente tarea. Es el guía quien toma las decisiones y conduce, en este caso asignado al momento de reunirse y de manera consensuada al participante con mayor experiencia, antes referido como viola 3. Las seguidoras, por su parte, buscaron estar junto y respirar con el guía. La sincronización expuso momentos claves, tales como los ataques iniciales y los finales o cierres de sonido así como la respiración o articulación del fraseo musical, dos cuestiones a su vez asociadas al uso del arco en la interpretación. Una de las participantes mencionó también los acentos o puntos culminantes de la frase, sin mayores especificaciones. No obstante, las tres músicas insistieron en la importancia de la atención y la comunicación continua y multimodal -sonora y gestual- porque siempre, puede ser minimamente distinto. En cuanto a la reinterpretación, explicaron la necesidad de sincronizar mejor las últimas cuatro notas.

\section{Discusión}

En este trabajo se investigó la construcción temporal musical conjunta en una situación de reinterpretación espontánea en fila de orquesta a partir de una instancia de ejecución fuera de programa. El análisis basado en la variabilidad de los intervalos de tiempo horizontal y vertical entre ataques de sonido se completó luego con las ideas y las estrategias informadas por las participantes acerca de su actividad y de la experiencia en particular.

Para ello, se abordó inicialmente el estudio de la variabilidad entre ataques de sonidos sucesivos, observándose diferencias entre versiones en términos de la velocidad promedio de ejecución y de la amplitud de variación del ITEA con independencia del valor de la unidad rítmica escrita asociada al evento, lo que llevó al interrogante y a la indagación acerca del comportamiento individual de ejecución en relación con el grupo.

Respectivamente, las diferencias entre mismos ITEA en una versión y en otra evidenciaron la activa participación de los músicos en el afán por alcanzar un toque al unísono, más allá de los roles líder/seguidor asignados. En efecto, los alargamientos y/o acortamientos de duración de los eventos dieron cuenta de los ininterrumpidos ajustes que las ejecutantes realizaron en función de la interpretación y de las eventuales variaciones que tuvieron lugar por parte de sus compañeros en el transcurso de la ejecución. A propósito, el alineamiento y las 
declaraciones de los músicos relativas a sus estrategias de coordinación y sincronización constataron la presencia del guía. No obstante, este último también realizó modificaciones, sea porque las promovió o sea porque -en razón de su experticia y de su rol relevante- respondió y acudió a compensar y a sincronizar para lograr la unidad. Cualquiera fuera el caso, la reinterpretación devino sutilmente nueva para las tres participantes y, en medio de los esfuerzos por aunarse en el conjunto, adquirieron especial relevancia el dinamismo y la flexibilidad.

Los variados movimientos temporales, sin embargo, presentaron reducción unánime entre versiones en los puntos de inflexión relativos a la estructura métrica de la música. La minimización de las variaciones expresivas, de hecho, recayó sobre la primera división de la pulsación -atenta a las particularidades del extracto musical con comienzo acéfalo- y la pulsación, con excepción del evento final, tendientemente más libre conforme el decaimiento y cierre de la sección y de la frase (Todd, 1985). La estabilidad asociada a la estructura en dichos puntos no necesariamente se correlaciona con una acentuación de tipo fenoménica rítmica o melódica (Lerdhal y Jackendoff, 1983). En razón de esto, la acentuación métrica pareciera marcar posibles espacios referenciales de ordenamiento para la interpretación temporal conjunta y/o de restablecimiento regular de la sincronización adaptativa a lo largo de la ejecución musical.

En términos generales, la sincronización entre las intérpretes fue elevada en ambas versiones, alcanzando puntos máximos sobre los emplazamientos estructurales de la división del tiempo y la pulsación, coincidentemente con los momentos de mayor estabilidad temporal expresiva en la ejecución. Al respecto, el evento 1 resultó notoriamente más sincronizado en la segunda versión que en la primera, y más asincrónicos los eventos próximos a las notas inicial y final, independientemente de la versión. No obstante, se observó un prevalente alineamiento de las ejecutantes detrás de la viola-guía 3 durante la segunda interpretación, acompañado de una sutil reducción del asincronismo medio entre las ejecutantes seguidoras, violas 1 y 2 , a la vez que de un mayor asincronismo de las mismas en relación a la voz de la guía, viola 3 , donde se alcanzaron valores superiores a los reportados por otros estudios (Rasch, 1979), indicando a su vez una diferencia de ordenamiento del alineamiento entre la primera y segunda versión.

De este modo, en acuerdo con las declaraciones de las participantes, la construcción temporal unívoca en fila de orquesta sugiere una acción continua e interdependiente de pequeñísimos ajustes y compensaciones momento a momento, donde la adaptación a la fase parece ordenarse en base al seguimiento de la voz del líder y la valoración del timing de cada una de las restantes fuentes implicadas a su vez en la voz del guía quien, de manera igualmente dinámica y colaborativa, participa activamente del objetivo en común. La eventualidad de la variabilidad, sin embargo, prevé marcas regulares de acote de la variabilidad horizontal y de 
maximización de la sincronización asociados a la estructura métrica -"acentos", en palabras de una de las participantes-a fin de facilitar la coordinación temporal y la atención a futuro entre las artistas.

En consecuencia, a la acomodación temporal interna y la variabilidad del tiempo musical expresivo se agrega el tiempo macro regular asociado a la métrica musical de permanente soporte para la ejecución conjunta, donde el alineamiento y el anclaje métrico sobre el cual se monta la variabilidad expresiva conviven, en tanto formas organizativas temporales para la sincronización y la articulación conjunta asociadas a la conducción del fraseo musical durante la ejecución.

Por último, dadas las diferencias de tempo, de variabilidad del ITEA y de alineamiento de las ejecutantes entre versiones coparticipativamente sincronizadas, es probable que la conformidad de las ejecutantes respecto de la reinterpretación se deba al hecho de haber alcanzado en la segunda interpretación una nueva versión, más alineada también, acorde a las intenciones compartidas que los dejó más satisfechos en cuanto a la expresión.

\section{Notas}

1. Juliette Epele: https://orcid.org/0000-0002-0258-2054

2. Isabel Cecilia Martínez: https://orcid.org/0000-0002-1837-5957

\section{Referencias}

Clarke, E. F. (2014). Lost and found in music: consciousness and subjectivity. Musicae Scienciae 18(3), 354-368. doi: 10.1177/1029864914533812

De Jaegher, H. y Di Paolo, E. A. (2007). Participatory sense-making: an active approach to social cognition. Phenomenology and the Cognitive Sciences, 6(4), 485-507. doi: 10.1007/ s11097-007-9076-9

Español, S. y Shifres, F. (2015). The artistic infant directed performance: a microanalysis of the adult's movements and sounds. Integrative Psychological and Behavioral Science, 49(3) 371-397. doi: 10.1007/s12124-015-9308-4

Goebl, W. y Palmer, C. (2009). Synchronization of timing and motion among performing musicians. Music Perception, 26, 427-438. doi: 10.1525/mp.2009.26.5.427

Goebl, W. y Parncutt, R. (2002). The influence of the relative intensity on the perception of onset asynchronies. Proceedings of the 7th International Conference on Music Perception and Cognition (ICMPC, 2002). Sydney, Australia. 
Epistemus - volumen 8 - número 1 (Julio de 2020)

DOI: https://doi.org/10.24215/18530494e 016

Goodman, E. (2002). Ensemble performance. En J. Rink (Ed.), Musical Performance: A Guide to Understanding (pp.153-167). Cambridge: Cambridge University Press.

Keller, P. E. (2008). Joint action in music performance. En F. Morganti, A. Carassa y G. Riva (Eds.), Enacting Intersubjetivity: A Cogntive and Social Perspective on the Study of Interactions (pp. 205-211). Amsterdam: IOS Press.

Keller, P. E. (2014). Ensemble performance: interpersonal alignment of musical expression. En D. Fabian, R. Timmers y E. Schubert (Eds.), Expressiveness in music performance: Empirical approaches across styles and cultures (pp. 260-282). Oxford: Oxford University Press.

Keller, P. E. y Appel, M. (2010). Individual differences, auditory imaginery, and the coordination of body movements and sounds in musical ensembles. Music Perception, 28(1), 27-46. doi: 10.1525/mp.2010.28.1.27

Large, E. W. y Palmer, C. (2002). Perceiving temporal regularity in music. Cognitive Science, 26(1), 1-37. doi: 10.1207/s15516709cog2601_1

Leman, M. (2016). The Expressive Moment. How Interaction (with Music) Shapes Human Empowerment. Cambridge, Massachusetts : MIT Press.

Lerdahl, F. y Jackendoff, R. (1983). A Generative Theory of Tonal Music. Cambridge, Massachusetts: The MIT Press.

Madison, G., Ullén, F. y Merker, B. (2017). Metrically structured time and entrainment. En M. Lesaffre, P. J. Maes y M. Leman (Eds.), The Routledge Companion to Embodied Music Interaction (pp. 22-30). Nueva York: Routledge.

Malloch, S. y Trevarthen, C. (Eds.). (2009). Communicative Musicality. Oxford: Oxford University Press.

Martínez, I. C. (2014). La base corporeizada del significado musical. En S. Español (Ed.) Psicología de la Música y del Desarrollo (pp. 71-110). Buenos Aires: Paidós.

Palmer, C. (1997). Music performance. Annual Review of Psychology, 48, 115-138. doi: 10.1146/annurev.psych.48.1.115

Rankin, S. K., Large, E. W. y Fink. P. W. (2009). Fractal tempo fluctuation and pulse prediction. Music Perception, 26(5), 401-413. doi: 10.1525/mp.2009.26.5.401

Rasch, R. A. (1979). Synchronization in performed ensemble music. Acustica, 43(2), 121 131.

Repp, B. H. (2010). Self-generated interval subdivision reduces variability of synchronization with a very slow metronome. Music Perception, 27(5), 389-397. doi: 10.1525/

mp.2010.27.5.389 
Shaffer, L. H. (1984). Timing in solo and duet piano performance. Quarterly Journal of Experimental Psychology, 36, 577-595. doi: 10.1080/14640748408402180

Sawyer, K. (2006). Group creativity: musical performance and collaboration. Psychology of Music, 34(2), 148-165. doi: 10.1177/0305735606061850

Tecumseh Fitch, W. (2012). The biology and evolution of rhythm: unravelling a paradox. En P. Rebuschat, M. Rohrmeier, J. A. Hawkins e I. Cross (Eds). Language and Music as Cognitive Systems (pp. 73-94). Oxford: Oxford University Press.

Timmers, R., Endo, S., Bradbury, A. y Wing, A. M. (2014). Synchronization and leadership in string quartet performance: a case study of auditory and visual cues. Frontiers in Psychology, 5(645). doi: 10.3389/fpsyg.2014.00645

Todd, N. (1985). A model of expressive timing in tonal music. Music Perception, 3(1), 33-57. doi: $10.2307 / 40285321$

Varni, G., Volpe, G. y Camurri, A. (2010). A system for real-time multimodal analysis of nonverbal affective social interaction in user-centric media. IEEE Transactions on Multimedia, 12, 576-590. doi: 10.1109/TMM.2010.2052592 